\title{
Structure in Minor-Closed Classes of Matroids
}

\author{
Jim Geelen, Bert Gerards and Geoff Whittle
}

\begin{abstract}
This paper gives an informal introduction to structure theory for minorclosed classes of matroids representable over a fixed finite field. The early sections describe some historical results that give evidence that well-defined structure exists for members of such classes. In later sections we describe the fundamental classes and other features that necessarily appear in structure theory for minor-closed classes of matroids. We conclude with an informal statement of the structure theorem itself. This theorem generalises the Graph Minors Structure Theorem of Robertson and Seymour.
\end{abstract}

\section{Introduction}

For the last thirteen years we have been involved in a collaborative project to generalise the results of the Graph Minors Project of Robertson and Seymour to matroids representable over finite fields. The banner theorems of the Graph Minors Project are that graphs are well-quasi-ordered under the minor order [34] (that is, in any infinite set of graphs there is one that is isomorphic to a minor of another) and that for each minor-closed class of graphs there is a polynomial-time algorithm for recognising membership of the class [32]. We are well on track to extend these theorems to the class of $\mathbb{F}$-representable matroids for any finite field $\mathbb{F}$.

It is important to point out here that day-to-day work along this track does not concern well-quasi-ordering or minor testing. The actual task and true challenge is to gain insight into the structure of members of proper minor-closed classes of graphs or matroids. The well-quasi-ordering and minor-testing results are consequences not necessarily easy ones - of the structure that is uncovered. Ironically, while one may begin studying structure with the purpose of obtaining marketable results, in the end it is probably the structural theorems themselves that are the most satisfying aspect of a project like this. To acquire that structural insight is the bulk of the work and the theorems that in the end describe the entire structure are the main deliveries of a project like this.

The point is related to topics discussed in two interesting articles by Tim Gowers $[15,16]$. The latter article deals, amongst other things, with the role played by "rough structure theorems" in combinatorics. From this perspective Szemeredi's Regularity Lemma can be regarded as a rough structure theorem for all graphs. The Regularity Lemma has become an indispensable tool and has been used to prove profound theorems. Of course there is no such thing as an all-purpose tool. As Gowers points out it tells us nothing that would enable us to find better bounds for Ramsey's Theorem. There is currently no tool, analogous to the regularity lemma, available for finding such bounds. He remarks in [15] that

A better bound seems to demand a more global argument, involving the whole graph, and there is simply no adequate model for such an argument in graph theory.

A little further on he is led to 
fantasise that there might be a sort of classification of red-blue colourings (or equivalently graphs) which would enable one to solve the problem ...

On reading both papers it seems clear that - stated in a language suited to the polemical purpose of this introduction - Gowers would like a rough structure theorem that gives the global structure of members of the class of graphs that have no fixed clique as an induced subgraph. We know from Ramsey's theorem that such graphs must have arbitrarily large holes, but it is an understanding of the global structure that would potentially give improved bounds.

Recall that a minor of a graph is obtained by deleting and contracting edges. If, instead of asking for the global structure of a graph with no induced $K_{n}$ for some fixed $n$, we ask for the global structure of a graph with no $K_{n}$ minor, then such a structure theorem already exists. That theorem is the Graph Minors Structure Theorem of Robertson and Seymour [33]. This theorem tells us that members of the class of graphs with no fixed $K_{n}$ minor are graphs that have a tree-like decomposition into graphs that are "essentially planar". Here essentially planar allows departure from planarity in three distinct but bounded ways where the bounds are a function of the size of the clique we are excluding. The Graph Minors Structure Theorem is truly the workhorse of the Graph Minors Project and it is hard to see how the other goals of the project could be achieved without it.

Early on Robertson and Seymour realised that their results were likely to be special cases of more general results for matroids represented over finite fields. If you are not familiar with matroids, then it suffices at this stage to be aware of the following. Any finite set of vectors in a vector space over a field $\mathbb{F}$ has an associated matroid $M$ that captures the key combinatorial properties of the collection. The matroids associated with such collections of vectors are the $\mathbb{F}$-representable matroids. Any graph $G$ has an associated matroid that is $\mathbb{F}$-representable for any field $\mathbb{F}$. There is a natural notion of substructure in matroids that we call "minor". Minors in graphs and matroids correspond in that the matroid associated with a minor of a graph $G$ is a minor of the matroid associated with $G$.

Let $q$ be a prime power. Robertson and Seymour conjectured - although apparently not in print - that the class of $G F(q)$-representable matroids is well-quasiordered, that is, that in any infinite set of such matroids there is one that is isomorphic to a minor. As said above we are well on track to prove this conjecture and also the conjecture that membership testing for minor-closed classes of $G F(q)$ representable matroids can be performed in polynomial time. Not that we have already laid out full proofs of these two conjectures, but we have achieved a structure theorem for minor-closed classes of $G F(q)$-representable matroids and we know how to apply that to well-quasi-ordering and membership testing. But, actually carrying out these applications, does require a full write-up of the proof of the structure theorem. That write-up will be laborious and so will take some time: the structure alone is already more involved for matroids than for graphs, and proving the Graph Minor Structure Theorem required two thirds of the 750 pages of the 23 Graph Minors papers. We add that, while it would be inconceivable to prove a structure theorem for matroids without having the Graph Minors Structure Theorem as a guide, the structural results that we have obtained are very far from being an incremental extension of the Graph Minors Project. 
We expect our structure theorem to have numerous applications other than wellquasi-ordering and minor testing. We mention one here. Rota [36] conjectured that for any finite field $\mathbb{F}$ there are a finite number of minor-minimal obstructions to representability over $\mathbb{F}$. Rota's Conjecture is probably the most famous conjecture in matroid theory. We expect that the structure theorem will prove an essential ingredient in a future proof of Rota's Conjecture.

Having said that, from now on we forget about applications and focus simply on the structure theory itself. The goal of this paper is not to give an explicit formal statement of a matroid minors structure theorem. That would involve technicalities and complications that would cloud the overall picture. Instead we adopt the following strategy. Sections 3, 4 and 5 present background material that gives evidence for the existence of structure in minor-closed classes of matroids. In Sections 6-10 we discuss topics that are necessary to an understanding of the structure theory. Structure is outside our control; it is what it is. The goal of these sections is primarily to make it clear why certain features necessarily play a role in a structure theorem. In Section 11, we present a very informal statement of the structure theorem itself. If, as a reader, you go away feeling that you have gained some insight into what this branch of structural mathematics is about we would be absolutely delighted.

\section{A matroid primer}

This paper does not require an extensive background in matroid theory, although it is unlikely that a complete matroid novice would get much from reading it. Terminology and notation generally follows Oxley's comprehensive text [28]. Oxley has also written a concise introduction to matroids [29]; an updated version of this paper is available on his website. In this section we cover some basic topics giving a slant that is relevant to a reading of this paper.

If $A$ is a matrix over a field $\mathbb{F}$ with columns indexed by a finite set $E$, then the column matroid of $A$ is the pair $M(A)=(E, r)$, where, for a subset $X$ of $E$, we have $r(X)$ is the rank of the set of columns indexed by $X$. It is easy to see that $M(A)$ satisfies the following:

(i) $0 \leq r(X) \leq|X|$ for each $X \subseteq E$;

(ii) $r(X) \leq r(Y)$ whenever $X \subseteq Y \subseteq E$;

(iii) $r(X \cup Y)+r(X \cap Y) \leq r(X)+r(Y)$ for all $X, Y \subseteq E$.

More generally, if $E$ is a finite set and $r: 2^{E} \rightarrow \mathbb{Z}$ is a function, then the pair $M=(E, r)$ is a matroid with rank function $r$ if the above properties hold. The set $E$ is the ground set of $M$ and the members of $E$ are the elements of $M$. The column matroids of matrices over $\mathbb{F}$ are called $\mathbb{F}$-representable or representable over $\mathbb{F}$.

It is almost certainly a rare property of a matroid to be representable over some field, yet, amazingly enough, it seems that this has yet to be proved [27]. But that is an aside, our interest here is primarily in representable matroids. The rank of a set of columns in a matrix is preserved under row operations and column scaling. It follows that column matroids are preserved under such operations. Readers of this paper with little prior knowledge of matroid theory could do worse than think of representable matroids as column labelled matrices where it is understood that 
two matrices are equivalent if one can be obtained from the other by row operations and column scaling. While it is certainly possible for two matroids that are not equivalent in the above sense to have identical column matroids it turns out that, for the problems that we discuss here, the distinction is not significant. (Nonetheless, in other contexts, so-called inequivalent representations of matroids are genuinely problematic and have been well studied in recent years [14].) Modulo this subtlety one can regard properties of a matrix that are invariant under row operations as being its matroidal properties. Note that row operations are just about the first thing that students learn to do in linear algebra so there are no surprises in the observation that many natural properties of matrices are matroidal.

Terminology for matroids is frequently inherited from matrices and vectors. For example, a set is independent if its rank is equal to its cardinality; otherwise it is dependent. A basis of a matroid $M$ is a maximal independent set. Readers with some familiarity with matroids may have seen them defined differently from here; most likely from their independent sets. It is a feature of the subject that matroids can be defined in many different, but equivalent, ways.

\section{Graphic matroids}

Other terminology is inherited from graph theory, for example a minimal dependent set is a circuit. The reason for this is that graphs also provide us with a fundamental class of matroids. Let $G=(V, E)$ be a graph and $\mathbb{F}$ be a field. A signed incidence matrix of $G$ is a matrix $A \in \mathbb{F}^{V \times E}$ where each column has exactly two nonzero entries, a 1 and a -1 in the rows labelled by its incident vertices (if the edge is a loop, then the column has only zeros). The matroid obtained is independent of the sign pattern and the field. It is called the cycle matroid of the graph $G$ and is denoted by $M(G)$. Note that the elements of the cycle matroid are the edges of the graph. A set of edges is independent in $M(G)$ if and only if it is the edge set of a forest in $G$. All matroids obtained in this way are called graphic.

At one extreme it may seem that cycle matroids do not capture much of the graph. Indeed all forests with edge set $E$ have the same cycle matroid, a rather trivial one where every set is independent. But with mild extra connectivity the picture changes quickly: a 3-connected graph can, up to the labels of the vertices, be recovered completely from its cycle matroid.

The fact that the elements of a graphic matroid correspond to edges of an associated graph creates a small wrinkle for the uninitiated. The vector space perspective makes it natural to think of the elements of a matroid as points, not edges. It's not hard to reconcile the perspectives of course. Let $A$ be a matrix obtained from a graph $G$ as described above. Let $B$ be a $|V| \times|V|$ identity matrix and consider the matrix $[B \mid A]$. Each element of the graphic matroid $M(G)$ lies in the span of a pair of elements of $B$. Thus $B$ plays a role akin to the vertices of $G$ and the elements of the cycle matroid are seen as naturally corresponding to edges of a graph. More generally we can see that any matroid with a basis $B$ having the property that all other elements are in the span of pairs of elements of this basis will have some sort of graphic structure. We'll return to this topic when we discuss frame matroids. 


\section{Minors and duality}

For a subset $D$ of $E$, the deletion of $D$ from $M$ is the matroid $M \backslash D=\left(E-D, r^{\prime}\right)$ where $r^{\prime}(X)=r(X)$ for all subsets $X$ of $E-D$. In the case that $M$ is the column matroid of a matrix $A$, then $M \backslash D$ is the column matroid of the matrix obtained by removing the columns labelled by $D$ from $A$. For a subset $C$ of $E$, the contraction of $C$ from $M$ is the matroid $M / C=\left(E-C, r^{\prime}\right)$, where $r^{\prime}(X)=r(X \cup C)-r(C)$ for all $X \subseteq E-C$. Geometrically contraction corresponds to projecting the vectors labelled by $E-C$ from the subspace spanned by $C$ onto a subspace that is skew to $C$. From a matrix perspective, if $M=M(A)$ for a matrix $A$, then we can obtain a representation of $M / C$ by first performing row operations so that the columns labelled by $C$ are in echelon form, then removing the rows that have nonzero entries in a column labelled by $C$, and finally removing the columns labelled by $C$. A minor of $M$ is a matroid of the form $M \backslash D / C$ for some disjoint subsets $C$ and $D$ of $E$. Minors form a natural partial order on matroids. A class of matroids is minor closed if every minor of a member of the class also belongs to the class. Evidently the class of $\mathbb{F}$-representable matroids is minor closed.

Given a matroid $M$ with ground set $E$, there is a unique matroid $M^{*}$ with ground set $E$ having the property that $B^{*}$ is a basis of $M^{*}$ if and only if $B^{*}=E-B$ for some basis $B$ of $M$. We say that $M^{*}$ is the dual of $M$. Evidently $\left(M^{*}\right)^{*}=M$. Moreover duality interchanges deletion and contraction in that $(M \backslash X)^{*}=M^{*} / X$ for any set $X$ of elements of $M$.

Matroid duality turns out to be perfectly natural in our fundamental examples. For example, if $M$ is represented over $\mathbb{F}$ by the matrix $A$, then $M^{*}$ is also $\mathbb{F}$-representable. Indeed, modulo appropriate labelling of the columns, a matrix $A^{\prime}$ represents $M^{*}$ if and only if the row spaces of $A$ and $A^{\prime}$ are orthogonal subspaces. It follows from this, that if $A$ is regarded as the generator matrix of a linear code, then the parity-check matrix of that code is a matrix representing $M^{*}$.

More surprising, and somehow truly fundamental, is the following fact. If $G$ is a graph, then the dual of the cycle matroid of $G$ is graphic if and only if $G$ is planar. Moreover, in the case that $G$ is a planar graph, then the dual of the cycle matroid of $G$ is the cycle matroid of $G^{*}$ where $G^{*}$ is any planar dual of $G$. Thus a matroid property defined in a purely combinatorial or algebraic way is seen to have a fundamental connection with a topological property. We will see more of this

phenomenon. It is one of the remarkable aspects of the theory of matroid minors and, indeed, of graph minors.

A matroid is called cographic if it has the form $M(G)^{*}$ for some graph $G$. Cographic matroids are sometimes called bond or cocycle matroids as the circuits of $M(G)^{*}$ are the bonds of $G$.

\section{Connectivity}

The connectivity function $\lambda_{M}$ of a matroid $M$ is defined, for all partitions $(A, B)$ of $E(M)$ by

$$
\lambda_{M}(A, B)=r(A)+r(B)-r(E(M))+1 .
$$

We usually abbreviate $\lambda_{M}(A, B)$ to $\lambda_{M}(A)$. The +1 is there solely to make graph and matroid connectivity align in a certain way and is often omitted. We say that 
$(A, B)$ is a $k$-separation of $M$ if $\lambda(A, B) \leq k$. If $(A, B)$ is a $k$-separation, then we say that $A$ is $k$-separating.

What is $\lambda_{M}$ measuring? Say that $M$ is represented as a collection of vectors in a vector space, and $(A, B)$ is a partition of $E(M)$, then $r(A), r(B)$ and $r(E(M))$ measure the dimension of the subspaces $\langle A\rangle,\langle B\rangle$ and $\langle E(M)\rangle=\langle A \cup B\rangle$ spanned by $A, B$ and $A \cup B$ respectively. In this case $\lambda(A)$ is one more than the dimension of the subspace $\langle A\rangle \cap\langle B\rangle$. The intuition is that, the higher the value of $\lambda(A, B)$, the more potential for communication there is between $A$ and $B$. At the bottom extreme we have $\lambda(A, B)=1$. In the represented cases, this means that $A$ and $B$ span skew subspaces so that the potential for mutual interaction is nonexistent.

We now consider the connection between graph and matroid connectivity. Let $G=(V, E)$ be a graph. For a set $X$ of edges of $G$, let $V(X)$ denote the set of vertices incident with edges in $X$. The connectivity function $\lambda_{G}$ of $G$ is defined for all partitions $(A, B)$ of $E$ by $\lambda_{G}(A, B)=|V(A) \cap V(B)|$. Evidently $V(A) \cap V(B)$ is a vertex cut that separates $A$ from $B$. Moreover, if $A$ and $B$ induce connected subgraphs, then $\lambda_{G}(A, B)=\lambda_{M(G)}(A, B)$. In the case that $A$ and $B$ do not induce connected subgraphs these values can differ significantly. Nonetheless it is still true to say that matroid connectivity generalises vertex connectivity. Of course this is another wrinkle for the uninitiated as, having got one's head around the fact that the elements of a graphic matroid are the edges of the graph, one might expect matroid connectivity to generalise edge connectivity, but that's not the case. It's definitely vertex connectivity that we capture most naturally in matroids.

\section{Some important matroids}

Certain matroids will turn out to be of considerable importance for us. Before we discuss these we need to acquaint ourselves with some elementary structures. An element $e$ of a matroid is a loop if $r(\{e\})=0$, equivalently $e$ is a loop if $\{e\}$ is dependent. A pair of elements $\{f, g\}$ of a matroid is a parallel pair if neither are loops and $\{f, g\}$ is dependent. This generalises graph terminology in that, if $M$ is the cycle matroid of a graph $G$, then the loops and parallel pairs of $M$ are precisely the loops and parallel pairs of $G$. A matroid is simple if it has no loops or parallel pairs. As in graph theory, for many purposes we can focus on simple matroids. Nonetheless, loops and parallel pairs are created by contraction so we allow their presence in general.

Let $q$ be a prime power. The $r$-dimensional vector space over $G F(q)$ is denoted $V(r, q)$. Evidently $V(r, q)$ is a matroid. From our point of view it contains some degeneracies in that the zero vector is a loop and two nonzero vectors on the same 1-dimensional subspace are a parallel pair. We obtain a canonical simple matroid by removing all but one element of each of the 1-dimensional subspaces. Via this, or one of a number of equivalent constructions, we obtain the projective geometry $P G(r-1, q)$. Note that, while $P G(r-1, q)$ has dimension $r-1$, regarded as a matroid it has rank $r$. If $M$ is a simple matroid representable over $G F(q)$ of rank at most $r$, then $M$ is isomorphic to a submatroid of $P G(r-1, q)$, that is, a matroid isomorphic to $M$ can be obtained by deleting points from $P G(r-1, q)$.

The role played by projective geometries in representable matroids is analogous to that played by cliques in graphs. Every simple rank- $r$ graphic matroid is a 
submatroid of the matroid $M\left(K_{r+1}\right)$. Note that a spanning tree in $K_{r+1}$ has $r$ edges so that $M\left(K_{r+1}\right)$ has rank $r$. Cliques and projective geometries control the number of elements in simple rank- $r$ graphic and $G F(q)$-representable matroids. In other words they control the growth rates of these classes. We'll return to this topic soon.

Let $M$ be a matroid obtained by randomly choosing an $n$-element set of vectors from a rank- $r$ vector space over an infinite field. The probability that $M$ has a dependent set of size less than $r+1$ is 0 , that is, every $r$-element subset of $E(M)$ is a basis. Put in matroid language, with a probability of 1 the matroid $M$ is the uniform matroid $U_{r, n}$. Of particular importance to us is the case $r=2$. Here the uniform matroid $U_{2, n}$ is just an $n$-point line. Note that $P G(1, q) \cong U_{2, q+1}$ so that no $G F(q)$-representable matroid can contain $U_{2, q+2}$ as a minor. A matroid is binary if it is representable over $G F(2)$. Evidently $U_{2,4}$ is not binary. It is a seminal - but not difficult - theorem of Tutte that the converse holds in that a matroid with no $U_{2,4}$-minor is binary. This is a striking example of the central theme of this paper. Matroids in general are wild, but the simple fact of excluding $U_{2,4}$ as a minor has put us into the highly structured class of binary matroids.

We don't always win this way. Let $G$ be a graph consisting of three edges, one a loop, the others forming a path of length 2 . If excluding $U_{2,4}$ imposes structure, surely excluding $M(G)$ must as well; if anything it's an even simpler matroid. But it's probably not so. It is widely believed that having an $M(G)$ minor is rare in that the proportion of $n$-element matroids with an $M(G)$-minor is asymptotically zero as $n$ tends to infinity. Notwithstanding this, Tutte's theorem is encouraging.

\section{Growth Rates of Minor-Closed Classes}

The growth rate of a class $\mathcal{M}$ of matroids is the function $f$, where $f(r)$ is the maximum number of elements in a simple rank-r member of $\mathcal{M}$, if that maximum exists. The class of all matroids does not have a well-defined growth rate, for example long lines give arbitrarily large simple rank- 2 matroids. However many natural minor-closed classes do.

The projective geometry $P G(r-1, q)$ has $\frac{q^{r}-1}{q-1}$ elements, so this is precisely the growth rate of $G F(q)$-representable matroids. We say that this class has a growth rate that is base-q exponential.

The clique $K_{r+1}$ has $\frac{r^{2}+r}{2}$ edges, so graphic matroids have polynomial, indeed quadratic, growth rates. There is a big jump between exponential and polynomial, let alone between exponential and quadratic. Kung [24] conjectured that there are no minor-closed classes with growth rates that are intermediate between exponential and quadratic. That's a remarkably large gap, but it turns out that this conjecture is true as we shall see later in this section. The picture that emerges when growth rates are studied provides clear evidence for structure in members of minor-closed classes.

In this section we consider growth rates in somewhat more detail. We know from graphs that significant jumps in growth rates can occur. We cannot resist giving the proof of the following theorem of Mader [25].

Theorem 3.1 (Mader's Theorem) Let $m$ be an integer greater than 2 . There is a constant $c_{m}$ such that, if $G=(V, E)$ is a graph with no $K_{m}$-minor, then $|E| \leq c_{m}|V|$. 
Proof Let $c_{m}=2^{m-3}$. We use induction on $m$. The case $m=3$ is easy. Assume $m \geq 4$. Let $u$ be a vertex of a minor-minimal graph $G=(V, E)$ satisfying $|E| \geq$ $2^{m-3}|V|$. By minimality there are more than $2^{m-3}$ edges incident with $u$ and every such edge is in at least $2^{m-3}$ triangles. Hence the graph $G_{u}$ induced by the vertices adjacent to $u$ has minimum degree at least $2^{m-3}$ and hence $\left|E\left(G_{u}\right)\right| \geq 2^{m-4}\left|B\left(G_{u}\right)\right|$. By induction $G_{u}$ contains a $K_{m-1}$-minor. The corresponding minor induced by $G_{u}$ and $v$ is a $K_{m}$-minor in $G$.

If $\mathcal{G}$ is any proper minor-closed class of graphs, then $\mathcal{G}$ avoids some graph $H$ and hence avoids a clique with as many vertices as $H$. It follows from Mader's Theorem that the growth rate of $\mathcal{G}$ is bounded by a linear function. An immediate consequence is that the growth rate of any proper minor-closed class of graphic matroids is bounded by a linear function.

The situation is in striking contrast to the case where we are avoiding $K_{m}$ as a subgraph. Evidently $(m-1)$-partite graphs cannot have a $K_{m}$ subgraph and these can have quadratically many elements. As is well known, Turán's Theorem [39] identifies the extremal examples and growth rate exactly in this case.

What is going on in the graph minors case? The sharp drop in growth rate shows that it is difficult for a graph to avoid a fixed graph $H$ as a minor. The graph must have low density and its minors must have low density also. The conclusion that avoiding $H$ as a minor imposes structure on a graph is inescapable. (This is with the benefit of hindsight, and hindsight gives 20-20 vision.) Of course, one way to avoid having $H$ as a minor is to be embeddable on a surface on which $H$ cannot be embedded. This is certainly explicit structure. Readers already familiar with the Graph Minors Structure Theorem of Robertson and Seymour [33] will be aware that this is not the full story, but it is the heart of the story. We'll give a fuller picture later. However note that, just as topology entered into the picture in a surprising way via the connection with planar graph duality, it has, once again, emerged as part of the answer after asking a purely combinatorial question.

Being embeddable on an appropriate surface is one way to avoid $H$. Here is another. Consider $K_{3, n}$. This is a graph that does not have $K_{5}$ as a minor and by making $n$ sufficiently large we can avoid embeddability on any surface that we like. Here we achieve the trick of avoiding $K_{5}$ by having too thin connectivity. This is another structural reason and we'll come back to that later as well.

Back to more general matroids. As noted earlier, a minor-closed class of matroids need not have a defined growth rate. But it turns out that once we exclude a line as a minor, then we do. Moreover, in the prime power case, we can characterise the extremal matroids. Kung [24] proved the following theorem. Recall that $U_{2, n}$ is the line with $n$ points.

Theorem 3.2 (Kung's Theorem) Let $q$ be a positive integer exceeding 1. If $M$ is a simple rank-r matroid with no $U_{2, q+2}$-minor, then $|E(M)| \leq \frac{q^{r}-1}{q-1}$. Moreover if equality holds and $r \geq 4$, then $q$ is a prime power and $M$ is the projective geometry $P G(r-1, q)$.

Here is part of the proof. A parallel class is a maximal set whose 2-element subsets are parallel pairs. Assume that $M$ has no $U_{2, q+2}$-minor. Choose an element $e \in E(M)$. The parallel classes of $M / e$ are easily seen to be the lines of $M$ containing 
$e$ with $e$ removed. By induction, there are at most $\frac{q^{r-1}-1}{q-1}$ of them. But each has at most $q$ elements, as otherwise $M$ has a $(q+2)$-point line as a restriction. Therefore $M$ has at most $q \frac{q^{r-1}-1}{q-1}+1=\frac{q^{r}-1}{q-1}$ elements as required. Identifying the extremal structures in the case that equality holds is also quite straightforward and given by Kung in [24].

Earlier we mentioned that Kung had conjectured that there were no growth rates for minor-closed classes in between quadratic and exponential. This was part of his Growth Rates Conjecture. Another part of his conjecture was that an analogue of Mader's Theorem held for matroids. This part was proved by Geelen and Whittle [13]. In that paper it is proved that, if $\mathcal{M}$ is a minor-closed class of matroids that does not contain $U_{2, n}$ for all $n \geq 2$ and does not contain all graphic matroids, then the growth rate of $\mathcal{M}$ is bounded by a linear function. Further progress on Kung's conjecture was made by Geelen and Kabell [11]. Eventually the full conjecture was proved by Geelen, Kung and Whittle [12].

Theorem 3.3 (Growth Rate Theorem) Let $\mathcal{M}$ be a minor-closed class of matroids. Then either,

(i) There exists an integer $c$ such that $|E(M)| \leq c(r(M))$ for all simple matroids $M \in \mathcal{M}$,

(ii) $\mathcal{M}$ contains all graphic matroids and there exists an integer $c$ such that $|E(M)| \leq$ $c(r(M))^{2}$ for all simple matroids $M \in \mathcal{M}$,

(iii) there is a prime-power $q$ and an integer $c$ such that $\mathcal{M}$ contains all $G F(q)$ representable matroids and $|E(M)| \leq c q^{r(M)}$ for all simple matroids $M \in \mathcal{M}$, or

(iv) $\mathcal{M}$ contains $U_{2, n}$ for all $n \geq 2$.

Let $\mathcal{U}(q)$ denote the class of matroid with no $U_{2, q+2}$-minor. We've already seen that $\mathcal{U}(2)$ is precisely the class of binary matroids. It follows from the Growth Rate Theorem that in general $\mathcal{U}(q)$ has essentially the same growth rate as the class of $G F\left(q^{\prime}\right)$-representable matroids where $q^{\prime}$ is the largest prime power less than or equal to $q$ and $\mathcal{U}(q)$ contains all matroids representable over a field of order at most $q$. But as $q$ grows, the $\mathcal{U}(q)$ zoo is seen to rapidly contain some pretty wild beasts. A notoriously wild class of matroids is the class of spikes; see for example [4, 20]. Spikes have no $U_{2,6}$-minor but have linear linear growth rate so have no impact on growth rates.

Incidentally, there are natural ways in which one can associate a matroid with a hypergraph. Doing this with, for example, 3-regular hypergraphs where each edge is incident with three vertices gives classes of matroids with cubic growth rates. It follows from the Growth Rate Theorem that these classes are not minor closed. Depending on the precise way that the associated matroid is defined, one inevitably builds either arbitrarily long lines or large projective geometries as minors of members of the class. 


\section{Frame Matroids and Varieties}

Kung gained the intuition for his Growth Rate Conjecture from joint work with Kahn [23] where they studied so-called varieties of matroids. We will describe this seminal work in this section as it gives insight into why certain classes of matroids necessarily play a role in matroid structure theory. Part (i) of the Growth Rate Theorem indicates that graphic matroids play a fundamental role, and that is clearly true, but there are certain somewhat richer classes with quadratic growth rates that necessarily play a role in structure theory. We begin by describing these classes.

We say that a matroid $M$ is a frame matroid if there exists a matroid $M^{\prime}$ with a basis $B$ having the property that $M=M^{\prime} \backslash B$ and that every element of $M^{\prime}$ is in the span of a pair of elements of $B$. Note that, if $M$ is a frame matroid, then so is the matroid $M^{\prime}$ described above; to see that simply add and delete a basis consisting of elements parallel to the elements of $B$. The point is that we allow, but do not require, the elements of $B$ to be part of our matroid. We have seen that graphic matroids are frame matroids. It is not hard to see that minors of frame matroids are frame matroids so that frame matroids form a minor-closed class of matroids.

Let $q$ be a prime power and $r$ a positive integer. We now construct an important class of frame matroids over $G F(q)$. We define them as submatroids of $P G(r-1, q)$. Let $B=\left(b_{1}, b_{2}, \ldots, b_{r}\right)$ be a basis of $P G(r-1, q)$. We lose no generality in saying that the elements of $B$ label the columns of an identity matrix. For each pair $i, j$ with $i<j$ add all distinct vectors having 1 in row $i$, a non-zero element in row $j$ and zeros in all other coordinates. There are $q-1$ such vectors for each choice of $i$ and $j$. Let $D(r, q)$ denote the submatroid of $P G(r-1, q)$ consisting of the elements we have described. It turns out that dependencies in $D(r, q)$ are determined purely by the structure of the multiplicative group $G F(q)^{*}$ of $G F(q)$. These matroids were studied by Dowling [3] who showed that one can begin with an arbitrary finite group $\Gamma$ and define a frame matroid $D(r, \Gamma)$ whose dependencies are determined by group multiplication. In the case that $\Gamma=G F(q)^{*}$ we have $D(r, \Gamma)=D(r, q)$.

These matroids can also be defined graphically. We can obtain a directed grouplabelled graph from our original construction as follows. Let $B$ label the vertices of our graph. For each pair $b_{i}$ and $b_{j}$ of vertices where $i<j$ we add $q-1$ edges directed from $b_{i}$ to $b_{j}$ and label these edges by the elements of $G F(q)^{*}$. Take a cycle in the graph and traverse it. Multiply the group values of the edges that have been traversed in the direction of the edge with the inverses of the group values of the edges that have been traversed against the direction of the edge. It is not hard to see that the cycle is a circuit in $D(r, q)$ if and only if the product is 1 . We can also see that the assignment of directions to edges was essentially arbitrary. If we reverse the direction of an edge we simply invert the group value. The procedure can be reversed of course and, beginning with a group-labelled directed graph, one can describe rules to obtain an associated matroid. If the graph is the one defined above, then the associated matroid is $D(r, q)$. Note that there are other matroids that can be associated with group-labelled graphs. The whole area is the subject of an extensive study by Zaslavsky; see for example [42].

Let $\Gamma$ be a finite group. We call a matroid that is a minor of $D(r, \Gamma)$ a Dowling matroid over $\Gamma$. Certainly Dowling matroids over $\Gamma$ are frame matroids, but we also have the stronger property that a rank- $r$ simple Dowling matroid over $\Gamma$ is 
a submatroid of $D(r, \Gamma)$. Thus, just as we have seen with graphic and $G F(q)$ representable matroids, each simple rank- $r$ member of the class is a submatroid of a canonical rank-r member of the class. Observe too that all members of the class of Dowling matroids over a group $\Gamma$ are representable over a field $\mathbb{F}$ if and only if $\Gamma$ is a subgroup of $\mathbb{F}^{*}$. Note also that $D(r, \Gamma)$ has $\left(\begin{array}{l}r \\ 2\end{array}\right)|\Gamma|+r$ elements so that the class of Dowling matroids has, as predicted by the Growth Rates Theorem, quadratic growth rate.

The multiplicative group of $G F(2)$ is the trivial group. In this case the associated class of Dowling matroids is precisely the class of graphic matroids. This is perhaps surprising since we have defined graphic matroids to be matroids on the edges of a graph, whereas $D(r, \Gamma)$ is a matroid on the edges and vertices of an appropriate graph. Indeed, if $\Gamma$ is the trivial group, then $D(r, \Gamma) \cong M\left(K_{r+1}\right)$. To see this, take the representation of $M\left(K_{r+1}\right)$ consisting of all columns with exactly two nonzero entries. The set of all edges incident with a fixed vertex of $K_{r+1}$ is a basis $B$ of $M\left(K_{r+1}\right)$. We may perform row operations to convert the columns labelled by $B$ into an identity matrix. Observe that every other edge is in a triangle with an edge in $B$, so that, in the new matrix, all other edges have exactly two nonzero entries, and the matrix we have is precisely the one obtained in the construction of $D(r, 2)$.

We now consider Kahn and Kung's theorem [23]. Let $M_{1}$ and $M_{2}$ be matroids on disjoint ground sets $E_{1}$ and $E_{2}$. Then the direct sum $M_{1} \oplus M_{2}$ is defined to be the matroid on $E_{1} \cup E_{2}$ with rank function $r_{M_{1} \oplus M_{2}}(X)=r_{M_{1}}\left(X \cap E_{1}\right)+r_{M_{2}}\left(X \cap E_{2}\right)$ for all $X \subseteq E_{1} \cup E_{2}$. It is elementary that $M_{1} \oplus M_{2}$ is in fact a matroid. If $X$ is a set of elements of the matroid $M$, then the restriction of $M$ to $X$, denoted $M \mid X$ is the matroid obtained by deleting the elements of $M$ not in $X$, that is, $M \mid X=M \backslash(E-X)$. It is easily seen that if $(A, B)$ is a partition of the ground set of the matroid $M$, then $M=M|A \oplus M| B$ if and only if $\lambda_{M}(A, B)=1$.

Let $\mathcal{M}$ be a minor-closed class of matroids that is closed under isomorphism. A sequence of universal models for $\mathcal{M}$ is a sequence $U_{1}, U_{2}, \ldots$ of matroids such that for all integers $r>0$, the matroid $U_{r}$ has rank- $r$ and such that each simple rank- $r$ matroid in $\mathcal{M}$ is a isomorphic to submatroid of $U_{r}$. We say that $\mathcal{M}$ is a variety if the following hold:

(V1) $\mathcal{M}$ is closed under direct sums, that is, if $M_{1}$ and $M_{2}$ are in $\mathcal{M}$, then so too is $M_{1} \oplus M_{2}$.

(V2) $\mathcal{M}$ has a sequence of universal models.

It is clear that if $G$ is a finite group, then the Dowling matroids over $G$ form a variety whose universal models are the Dowling geometries $D(r, G)$ and if $q$ is a prime power, then the class of $G F(q)$-representable matroids forms a variety whose universal models are the projective geometries over $G F(q)$. Kahn and Kung [23, Theorem 14] prove that, apart from three classes that are degenerate due to extremely low connectivity, these are the only varieties.

Kahn and Kung's theorem is certainly a strong theorem. It gives insight into why one would expect projective geometries and Dowling geometries to play a role in structure theory. Nonetheless, one may ask why the condition (V1) is imposed? The condition that we are closed under direct sums seems to be relatively mild but it is vital as otherwise the theorem does not hold. If we remove that condition we 
uncover many more minor-closed classes with sequences of universal models. Some of these have degeneracies due to low connectivity. It is natural to believe that the others are in some sense closely related to varieties. Indeed one can conjecture that they are obtained by "perturbing" varieties in ways related to perturbations discussed later in this paper. There are many interesting open problems in this area.

\section{Regular Matroids}

Growth rates and varieties give us evidence for structure from a very general perspective. Evidence comes from quite a different direction when we see explicit structural characterisations of specific minor-closed classes of matroids. Most strikingly, Seymour [37] gave such a characterisation for regular matroids. In this section we discuss that characterisation.

A matrix $A$ over the rationals is totally unimodular if the determinant of every square submatrix of $A$ is in $\{0,1,-1\}$. A matroid is regular if it can be represented by a totally unimodular matrix. For a variety of reasons regular matroids are one of the most well-studied classes of matroids. Tutte [41] proved that a matroid is regular if and only if it is representable over all fields and gave an excluded-minor characterisation of the class. Graphic and cographic matroids are regular. Moreover, Heller [17] proved that the growth rate of regular matroids is precisely that of the class of graphic matroids. Apart from that the class remained poorly understood and many questions remained open for totally-unimodular matrices and regular matroids until Seymour proved his Regular Matroid Decomposition Theorem [37], to date probably the most famous theorem in matroid theory. This theorem gives a precise structural characterisation of regular matroids. We'll begin by stating a consequence of it.

Let $(A, B)$ be a partition of $E(M)$ for some matroid $M$. Recall that $(A, B)$ is a 3 -separation if $\lambda(A, B) \leq 3$. In general a regular matroid need not be either graphic or cographic. But, as an immediate consequence of Seymour's theorem we obtain

Corollary 5.1 Let $M$ be a 3-connected regular matroid with at least 11 elements having the property that, whenever $(A, B)$ is a 3-separation of $M$, then either $|A| \leq 5$ or $|B| \leq 5$. Then $M$ is either graphic or cographic.

Thus, whenever we impose a reasonably mild connectivity condition we fall into one of two fundamental subclasses. What happens when we fail that connectivity test? Then there must exist a 3 -separation that splits the matroid in some relatively serious way. But, in this case, we can perform a standard matroid decomposition operation to obtain two smaller matroids, both of which are minors of the original matroid. This process can be repeated until we obtain pieces that are either graphic, cographic or copies of a certain 10-element matroid. Moreover, the original matroid can be canonically built from the pieces. The decomposition and composition operations are not difficult to describe - they are generalisations of the clique-sum operations for graphs - but the details need not concern us here.

Seymour's Theorem is a remarkable result. It is an exact structural description of regular matroids. It provides a road map for proving theorems about regular matroids; if a property holds for graphs, cographs and is preserved under certain elementary sums, then it holds for regular matroids. Moreover it leads to a polynomial- 
time algorithm for recognising regular matroids [40]. We could not ask for more from a structure theorem.

Seymour's theorem is extremely suggestive. Regular matroids form a proper minor-closed class of binary matroids. Highly connected regular matroids must either be graphic or cographic. We can fantasise that regular matroid decomposition is indicative of structure for any proper minor-closed class of binary matroids; or $G F(q)$-representable matroids for that matter. What hope is there for such a fantasy?

Life rapidly becomes more complicated in more general minor-closed classes. It's not hard to see that regular matroids are, in essence, the matroids obtained by excluding $P G(2,2)$. What happens if we are in the class obtained by excluding a larger projective geometry, say $P G(10,2)$ ? This class contains all graphic matroids of course and, by the Growth Rates Theorem, it has quadratic growth rate. If structure is there, graphic matroids should play a fundamental role. Is this the case? Consider an example. Construct a matroid as follows. Take the cycle matroid of a large clique represented by columns with exactly two nonzero entries. Now add eight arbitrary "junk" rows to the matrix and consider the matroid $M$ represented by the columns of the matrix $A$ we have constructed. Removing a row from $A$ corresponds to first adding a vector $v$ with a 1 in that row and zeros elsewhere, and then contracting $v$ from the extended matroid. We see that we can make $M$ graphic after a sequence of eight such operations. This is clearly a property preserved under minors. But it is not possible to make $P G(10,2)$ graphic after a sequence of eight such operations. We have shown that our matroid $M$ does not have a $P G(10,2)$ minor. The junk rows ensure that $M$ is unlikely to be graphic and it is clearly not possible to decompose $M$ across small separations.

This will come as no surprise to readers familiar with the Graph Minors Structure Theorem. The role played by the junk rows is analogous to the role played by apex vertices in that theorem. Just as we can add only a bounded number of apex vertices we would expect to be able to add only a bounded number of junk rows. The upshot is clear. In general we of course have to let go of having graphic or cographic as the core objects of our structure. Instead the best we could hope for are objects that are in some sense close to being graphic or cographic.

\section{Modularity}

What about the role played by 3 -separations in regular matroid decomposition? In fact there really is something special about 3-separations that persists for more general classes of binary matroids. Consider an example. Choose a binary matroid with a partition $(A, B)$ such that $M \mid A \cong M(G)$ for some large graph $G$. Say that $(A, B)$ is a $t$-separation. Recall that this means that $\lambda(A, B)=r(A)+r(B)-r(M)+$ $1 \leq t$. Assume that $\langle B\rangle \cap A \cong M\left(K_{t}\right)$. We hope this makes sense. The picture to have in mind is that of $M$ consisting of the cycle matroid of a large graph where the space of interaction with the rest of the matroid $B$ is spanned by the relatively small subclique $M\left(K_{t}\right)$ of $A$. The question we ask is "Can we build a minor containing $A$ that is incompatible with the graphic structure we now see on $A$ ?" Effectively we are allowed to contract elements from $B$ so long as we always contract elements outside the span of $A$. 
What if $(A, B)$ is a 3 -separation, so that $\lambda(A, B) \in\{1,2,3\}$ ? In the case that $\lambda(A, B)=1$, we have a direct sum and nothing we do to $B$ affects the structure of $A$. Say $\lambda(A, B)=2$, then we have a single edge $e$ in $K_{2}$ and all that we can do from $B$ is to produce other edges in parallel with $e$. Say $\lambda(A, B)=3$. Then the matroid $M\left(K_{3}\right)$ is a 3-point line and, in binary space, this is a full line. Again, operations from $B$ can only put points in parallel with existing points. Thus, for 3 -separations, nothing we do from $B$ can perturb the graphic structure that we see in $A$.

What if $\lambda(A, B)=4$ ? Here $M\left(K_{4}\right)$ has rank-3 and spans a plane in the underlying projective space. The points on this plane form a copy of $P G(2,2)$, the well-known Fano plane. This plane has seven points, but $K_{4}$ has only six edges, so there is a point $p$ not occupied by the points of $M\left(K_{4}\right)$. If it is possible to project an element of $M$ onto $p$ then we have obtained a minor that conflicts, albeit in a small way, with the graphic structure we have for $G$. Now imagine that $B$ is not the only such set "poking out" of $A$. Then maybe it would be possible to accumulate the evidence of such incompatibility and build a serious conflict with the graphical structure of $G$. How one accumulates such evidence is precisely what one needs to think about in proving structure theorems.

What if it is not possible to project an element of $B$ onto $p$ ? Then our copy of $M\left(K_{4}\right)$ is a so-called "modular flat". In this case we have no conflict with the graphical structure of $A$. It turns out to be a consequence of the modularity of $M\left(K_{4}\right)$ that the graphical structure we have found for $G$ can be enlarged and extended into $B$ - not necessarily all the way as there may be 3 -separations within $B$. So a 4-separation will either give us evidence for lack of graphic structure or an opportunity to enrich our graphic structure.

Now assume that we have extended the graphic structure of $G$ as far as possible into the rest of the matroid. It follows from the above discussion that we may have arbitrarily many 3 -separating sets glued onto $A$, but each higher-order $k$-separating set glued onto $A$ will be providing us with evidence for lack of graphic structure, and this cannot happen in an uncontrolled way without building a significantly richer non-graphic minor.

The crucial observation was that the modularity of $M\left(K_{4}\right)$ controlled the structure of life in $B$. The fact that modularity has this effect is further evidence for the existence of structure and we now consider the topic of modularity in more detail. A flat $F$ of a matroid $M$ is modular if $r(X \cap F)+r(X \cup F)=r(X)+r(F)$ for all flats $X$ of $M$. Recall that contraction of an element in a matroid corresponds to projection from that element. Let $F$ be a modular flat of a matroid and $x$ be an element outside of $F$. It may be that $F$ is no longer a flat in $M / x$ as some element $z$ is captured in the span of $F$ in $M / x$. It follows from the definition of rank in the contraction that, in this case $r(F \cup\{x, z\})=r(F)+1$. Let $Z$ be the flat spanned by $\{x, z\}$. Then $r(Z)=2$ and, by the modularity of $F$ we have $r(Z \cap F)=1$. Thus there is a point $z^{\prime} \in Z \cap F$ and the effect of contracting $x$ is to do nothing more than add $z$ in parallel with $z^{\prime}$; not a particularly exciting event. The same argument applies when we contract any set $X$ of elements that is skew to $F$, that is $r(F \cup X)=r(F)+r(X)$. Hence modular flats are precisely those flats that cannot be enriched by contraction of elements outside their span.

As observed above, modularity imposes significant structure on a matroid modulo reasonable connectivity assumptions. Assume that the matroid $M$ is 3-connected 
and $M$ has a modular flat $F$. It is not difficult to prove that if $M \mid F$ is isomorphic to the 3-point line $U_{2,3}$, then $M$ must be binary. Assume that $M\left(K_{4}\right)$ is modular in $M$. Again we easily see that $M$ must be binary. Moreover, if we raise the connectivity a little and assume that 3-separations have the property that one side has at most three elements, then we obtain the very strong consequence that $M$ must be graphic. This follows from a theorem of Seymour characterising when there is no circuit containing a given 3-element set in a binary matroid [38]. As observed above, this result gives us a powerful tool for demonstrating whether we have a matroid that is close to being graphic or whether we can produce something richer as a minor.

In binary matroids we would expect from the Growth Rates Theorem that significant extra richness implies that we can build increasingly large projective geometries as minors. For other fields that is not the case as we have the intermediate classes of Dowling matroids to consider. These have quadratic growth rates, but are significantly richer than graphic matroids. We now consider the same question as that considered at the start of this section, only this time we may assume that $M \mid A$ is a Dowling, rather than a graphic matroid. Consider a specific case. Assume that we are in the class of $G F(3)$-representable matroids. Here the class of Dowling matroids over the 2-element group is a class of $G F(3)$-representable matroids. As before we would like to be able to extend our Dowling structure into the set $B$ or get evidence from $B$ that there is richer structure in our matroid that is incompatible with the Dowling structure we have obtained. Our analogues of cliques are the Dowling geometries $D(r, 3)$. If $r=2$, then $D(r, 3)$ is the 4-point line and this is modular in any $G F(3)$-representable matroid, so, as before, we have to live with 3-separations. What about $r=3$ ? Here a genuine unpleasantness arises. The matroid $D(3,3)$ has representation.

$$
D=\left[\begin{array}{rrrrrrrrr}
1 & 0 & 0 & 1 & 1 & 0 & 0 & 1 & 1 \\
0 & 1 & 0 & 1 & -1 & 1 & 1 & 0 & 0 \\
0 & 0 & 1 & 0 & 0 & 1 & -1 & 1 & -1
\end{array}\right]
$$

It turns out that are several copies of $M\left(K_{4}\right)$ sitting inside $D(3,3)$. Indeed the matroid represented by the last six columns of $D$ is isomorphic to $M\left(K_{4}\right)$. Now imagine that the partition $(A, B)$ of our matroid $M$ has the properties that $\lambda(A, B)=4$, that $M \mid A$ is a ternary Dowling matroid with a restriction isomorphic to $D(3,3)$, and that the $M\left(K_{4}\right)$ restriction of $D(3,3)$ we have just described is extended to the cycle matroid of a large clique given by the set $A$. In other words, on one side of $(A, B)$ we see graphic structure, on the other side we see Dowling structure. It is a straightforward fact about Dowling matroids that, while $M\left(K_{4}\right)$ can be embedded in ternary Dowling matroids in a curious way, higher order cliques cannot. It follows that we cannot extend the Dowling structure of $B$ into the larger matroid. We have produced a matroid in which $D(3,2)$ is modular, but in which the Dowling structure of $D(3,2)$ is not extendable.

In summary we see that for binary-matroid structure there really is something special about 3-separations, just as with regular matroids. But for larger fields that specialness extends - at least - to certain types of 4-separations. 


\section{Branch Width and Tangles}

We gain insight into structure from considering growth rates and varieties partly because we slip past degeneracies caused by low connectivity. We saw a similar situation for regular matroids in Corollary 5.1 where, by insisting that matroids be sufficiently connected, we were able to obtain an extremely simple outcome for the structure theorem. There are two ways in which low connectivity affects structure. It can happen that we have many low order separations and it is possible to decompose the matroid in a tree-like way across these separations. If this does not occur, then we have regions of high connectivity separated from each other by lower order separations. We are able to identify such regions of high connectivity through the notion of a "tangle".

We say that a tree is cubic if all of its internal vertices have degree 3. A branch decomposition of a matroid $M$ is a cubic tree $T$ whose leaves are labelled by the elements of $M$. Each edge of this tree induces a partition of the ground set of $M$, that is, it induces a separation of a certain order. The width of the branch decomposition is the maximum order of a separation induced by an edge of $T$. The branch width of $M$ is the minimum width over all branch decompositions of $M$.

One can use the connectivity function of the graph to produce an analogous definition of the branch width of a graph. It turns out that, while there are differences between the connectivity function of a graph and a matroid, these differences are unimportant in that, apart from certain trivial exceptions, the branch width of a graph and its cycle matroid are the same [18]. Some readers may be more familiar with the related concept of tree width. While tree width does generalise to matroids [21], branch width is the more natural concept for matroids. Moreover, as we now explain, from a qualitative point of view, the two concepts are effectively equivalent.

A class of matroids has bounded branch width if there is a $k$ such that all members of the class have branch width at most $k$. It is known that a class of matroids has bounded branch width if and only if it has bounded tree width. If $\mathcal{M}$ is a class of matroids of bounded branch width, then we have an entirely satisfactory structural outcome for members $\mathcal{M}$. All members decompose across small separations into tree-like structures. There is now a huge literature on bounded tree width for graphs and we will not delve into that. The main point here is that, for matroids, most of the key wins that bounded tree width gives for graphs extend to classes of $G F(q)$-representable matroids of bounded branch width. For example such classes are well-quasi-ordered [7]. Also Hliněný [19] has extended Courcelle's Theorem [1] to such classes. Hliněný has shown that problems that can be stated in a certain logic have a polynomial-time algorithm for $G F(q)$-representable matroids of bounded tree width. The precise logic is not important here; the point is that, just as with graphs, many natural problems that are NP-complete for $G F(q)$-representable matroids in general can be stated in this logic. It follows that, in the case of $G F(q)$-representable matroids, the property of having a tree-like decomposition across small separations is an extremely powerful controller of the complexity of the underlying matroid. We also know that there are only a finite number of excluded minors for $G F(q)$ representability that have branch width at most $k$ for any positive integer $k$.

The upshot is that, from the point of view of matroid minors, for matroids representable over finite fields, classes of bounded branch width are easily dealt 
with. Note that this is not the case if we step outside that cosy world. For more general matroids bounding branch width does not seem to give us any gains. This is an appropriate time to take stock and refocus. It is conceivable that deep structural theorems exist for very general classes of matroids, but these theorems will not be constructive and will not give us algorithmic consequences. To illustrate the point consider the class of matroids representable over the rationals. One may perhaps expect this class to be reasonably well behaved. We are after all dealing with finite structures coordinatisable over a familiar field. But from the perspective of the problems we are interested in this is an intractable class. For example it's easy to construct infinite antichains of $\mathbb{Q}$-representable matroids of branch width 3. See also $[20,26]$ for other examples that illustrate just how radically things change here. That transition is a fact we have to accept.

We can now focus on classes of matroids of unbounded branch width. A matroid with very high branch width need not be highly connected in any strict sense of the word as it may have many low order separations; rather it must have regions of high connectivity. In [31] Robertson and Seymour make the notion of a region of high connectivity precise through the concept of a "tangle". Tangles are generalised to matroids in [10]. A basic problem of identifying an area of high connectivity in a graph or matroid is one of erosion. It's not possible to see such a region as a subgraph or submatroid without damaging valuable information. As an example from graphs, consider the road map of the United Kingdom. One highly connected region will correspond to Manchester. It's certainly not possible to find low order separations that split Manchester in any serious way. But if we attempt to isolate Manchester as a subgraph we will lose vertices and edges outside the city limits that the good citizens will tell you are valuable for gaining connectivity. How often do we hear comments like "I would never drive through town to get to my Aunt's place, rather I drive via X so that I can bypass the congestion on Y St." We might think that being able to take minors helps, but the problem persists.

Instead of trying to isolate a highly connected region as a substructure Robertson and Seymour had the following idea. If we claim that a region of the structure has connectivity at least $k$, then we are making the claim that it is not possible to split that region in any serious way with a separation of order less than $k$. Given a separation $(A, B)$ of order less than $k$ one should be able to unambiguously say whether the region lies mainly in $A$ or $B$. For example, if $(A, B)$ is a 50-separation of our road map, then it's unlikely that this separation could split Manchester in any serious way, so that most of Manchester is, say, unambiguously in $B$. It might be that most of London is in $A$, but, from the point of view of capturing Manchester we would think of $B$ as the "big" side. This ability to unambiguously decide on an ordering into big and small sides relative to a region of high connectivity for each small separation is precisely what we need and we can describe it axiomatically as follows.

A tangle $\mathcal{T}$ of order $k$ in a matroid $M$ is a collection of separations of order at most $k-1$ having the following properties.

(i) For each separation $(X, Y)$ of $M$ of order less than $k$, exactly one of $(X, Y)$ or $(Y, X)$ is in $\mathcal{T}$.

(ii) If $\left(A_{1}, B_{1}\right),\left(A_{2}, B_{2}\right)$ and $\left(A_{3}, B_{3}\right)$ are in $\mathcal{T}$, then $A_{1} \cup A_{2} \cup A_{3} \neq E(M)$. 
(iii) $(\{e\}, E(M)-\{e\}) \notin \mathcal{T}$ for each element $e$ of $M$.

If $(A, B) \in \mathcal{T}$, then we think of $B$ as the "big" side. Property (iii) is a non-triviality condition. Property (ii) says that $E(M)$ is not the union of three small sides and is a strengthening of the condition that we have an unambiguous small side. Assume that we fail that condition. Construct a cubic tree with one vertex of degree 3 and leaves labelled by $A_{1}, A_{2}$ and $A_{3}$. We have started to build a branch decomposition of order $k-1$. Evidently we have not found an obstruction to branch width $k$. But with (ii) we have indeed found such an obstruction and it turns out that tangles are precisely dual to branch width in that a matroid has branch width at most $k$ if and only if it has no tangles of order $k$. This is proved for graphs in [31] and extended to matroids in [10].

If $\mathcal{T}$ is a tangle of order $k$ in $M$ and $k^{\prime}<k$, then it is easily seen that we obtain a tangle $\mathcal{T}^{\prime}$ of order $k^{\prime}$ by simply restricting attention to the separations of order at most $k^{\prime}-1$ in $\mathcal{T}$. We say that $\mathcal{T}^{\prime}$ is a truncation of $\mathcal{T}$. A tangle is maximal if it is not a truncation of any other tangle. The question arises as to how the maximal tangles can interact. Anyone who has considered a modern conurbation will be aware of the issue. But it turns out that the answer is remarkably simple and the maximal tangles can indeed be disentangled. If $\mathcal{T}$ and $\mathcal{T}^{\prime}$ are distinct maximal tangles of order $k$ and $k^{\prime}$ respectively then there exists a separation $(X, Y)$ of order less than $\min \left\{k, k^{\prime}\right\}$ such that $(X, Y) \in \mathcal{T}$ and $(Y, X) \in \mathcal{T}^{\prime}$. Such a separation is a distinguishing separation. A tree decomposition of $E(M)$ is a tree $T$ whose vertices label a partition of $E(M)$. Note that each edge of a tree decomposition of $E(M)$ induces a separation of $M$.

Theorem 7.1 Let $\left\{\mathcal{T}_{1}, \mathcal{T}_{2}, \ldots, \mathcal{T}_{n}\right\}$ be the maximal tangles of a matroid $M$. Then there is a tree decomposition of $M$ with vertex set $\{1,2, \ldots, n\}$ such that the separation induced by and edge $\{i, j\}$ is a minimal-order distinguishing separation for $\mathcal{T}_{i}$ and $\mathcal{T}_{j}$.

Thus we can think of the matroid as being built from its maximal tangles in a tree-like way. In fact Theorem 7.1 holds for any set of tangles of $M$ that are incomparable in the truncation order. We discussed regular-matroid decomposition as a possible model for more general structure in minor closed classes. Note that regular-matroid decomposition is, essentially, decomposition relative to a maximal set of incomparable tangles of order at most 3.

From the tree of tangles we learn that the real task is to understand the structure of a matroid relative to a fixed high order tangle. The presence of other tangles certainly complicates the analysis and leads to a number of technical issues for proofs and so on, but, to gain insight into what is going on for the purposes of this paper we are well served by focussing on the case where $M$ is highly connected in the sense that it has a single high-order maximal tangle.

\section{Grids}

From now on, we may assume that matroids we are dealing with have arbitrarily high branch width, in other words we are dealing with matroids that contain arbitrarily high order tangles. The question is "What does high branch width give us?" and the answer is "It gives us grid minors". 
An $n \times n$ grid is a planar graph $G_{n}$ with vertex set $\{(i, j): 1 \leq i, j \leq n\}$ where vertices $(i, j)$ and $\left(i^{\prime}, j^{\prime}\right)$ are adjacent if and only if $\left|i-i^{\prime}\right|+\left|j-j^{\prime}\right|=1$. The branch width of a grid is an increasing function of $n$; indeed $M\left(G_{n}\right)$ contains a tangle of order $n$. It is also straightforward to prove that if $H$ is a planar graph, then $H$ is a minor of a sufficiently large grid. To see this, imagine drawing $H$ with slightly thick edges on fine graph paper. Robertson and Seymour prove that a graph with sufficiently large tree width has a large grid minor [30]. This theorem is extended to matroids in [9]. The matroid $U_{q, q+2}$ is the dual of the $U_{2, q+2}$, the $(q+2)$-point line.

Theorem 8.1 (Grid Theorem for Matroids) There is a function $f(n, q)$ such that, if $k \geq f(n, q)$ and $M$ is a matroid of branch width at least $k$, then $M$ has either $M\left(G_{n}\right), U_{2, q+2}$ or $U_{q, q+2}$ as a minor.

We have seen that $U_{2, q+2}$ is not $G F(q)$-representable. Since representability is closed under duality, neither is $U_{q, q+2}$. We immediately obtain the next corollary.

Corollary 8.2 Let $q$ be a prime power. Then, for all $n$ there exists $k$ such that, if $M$ is a $G F(q)$-representable matroid of branch width at least $k$, then $M$ has an $M\left(G_{n}\right)$-minor.

In other words, for $G F(q)$-representable matroids we can force arbitrary grids as minors by making the branch width sufficiently large. Using the observation that any given planar graph is a minor of a sufficiently large grid, the contrapositive of Corollary 8.2 says

Corollary 8.3 Let $q$ be a prime power and $H$ be a fixed planar graph. Then the class of $G F(q)$-representable matroids with no $H$-minor is a class of bounded branch width.

This is the structure theorem for excluding the matroid of a planar graph. To avoid a planar graph we must decompose in a tree-like way across bounded size separations. As planar graphs themselves form a class of unbounded branch width, the converse of Corollary 8.3 also holds. It follows from Corollary 8.3 and the fact that minor-closed classes of $G F(q)$-representable matroids of bounded branch width are well-quasi-ordered, that any minor-closed class of $G F(q)$-representable matroids that does not contain all planar graphs is well-quasi-ordered.

We mentioned earlier that topological properties can arise in unexpected ways. Even within graphs it's surprising the way that topology asserts itself; for matroids even more so. Imagine an intelligent life form based on the operating system of a computer. One could believe that their natural geometry was binary geometry and the natural objects of study binary matroids. Our creatures would discover branch width as a controller of complexity and would discover cycle matroids of planar graphs as the unique minimal minor-closed class of binary matroids having unbounded branch width. Planarity is an unavoidable part of the picture. Not only that, as we shall soon see, they will also be forced to eventually discover more general surface embeddings.

The Grid Theorem for Matroids is quite general in that it is not just a theorem for representable matroids. But having said that, it is not completely satisfactory. Ideally all the outcomes should be matroids of increasing branch width and lines 
and their duals do not have this property. Let $G$ be a graph. Embed the vertices of $G$ as a simplex in real space. Regard each edge of $G$ as a point placed freely on the line joining its incident vertices. The real-represented matroid formed by these points is the bicircular matroid of $G$, denoted $B(G)$. Cycle matroids of grids are essentially self dual. This is not the case with bicircular matroids of grids. The next conjecture is due to Robertson and Seymour, although not in print.

Conjecture 8.4 For all $n$ there exists $k$ such that if $M$ is a matroid with branch width $k$, then $M$ has a minor isomorphic to one of $U_{n, 2 n}, M\left(G_{n}\right), B\left(G_{n}\right)$, or $B\left(G_{n}\right)^{*}$.

Apart from very small values of $n$ the minors excluded by this conjecture form an antichain. Moreover, as $n$ grows the branch width of these matroids increases. Hence, if true, the conjecture is best possible. We believe Conjecture 8.4, but it is likely to be difficult to prove.

Finally we note that, as well as the original proof of the grid theorem for graphs given in [30], there are two other published proofs $[2,35]$. The proof given in [2] is particularly intuitive. However none of the proof techniques extend to matroids in any natural way. The proof for matroids given in [9] uses quite a different strategy.

\section{Core Classes and Deviations}

Let $q=p^{k}$ be a prime power and let $\mathcal{M}$ be a minor-closed class of $G F(q)$ representable matroids. We are gradually converging towards a statement of the essential features of a structure theorem for members of $\mathcal{M}$. Inevitably there will be layers to the structure depending on what we exclude. The Grid Theorem has already shown us what is, essentially, the bottom layer. If $\mathcal{M}$ does not contain all planar graphs, then $\mathcal{M}$ is a class of bounded branch width. Thus we may assume that $\mathcal{M}$ contains all planar graphs.

If our interest is in graphs, there is only one more layer to the structure. A proper minor-closed class $\mathcal{G}$ of graphs cannot contain all cliques so that there is an integer $n$ such that $K_{n}$ does not belong to $\mathcal{G}$. This situation is handled by the Graph Minors Structure Theorem. In this case, as observed in the introduction, graphs in the class have a tree-like decomposition into parts that are "essentially planar", where essentially planar allows bounded deviations from planarity in three specific ways. Thus we can say that the "core class" for the Graph Minors Structure Theorem is the class of planar graphs. In this section we first describe the core classes that arise in structure theory for our minor-closed class $\mathcal{M}$. We'll then discuss the issue of the deviations from the core classes.

Core classes. Consider the analogous question for the structure theorem for our class $\mathcal{M}$ of $G F(q)$-representable matroids. What are the core classes we obtain here? The existence of Dowling geometries and projective geometries guarantee that there will be more layers to the hierarchy for matroids. We know from the Growth Rate Theorem that if $\mathcal{M}$ is a class of matroids that does not contain the cycle matroids of all cliques, then, just as in the graph case, $\mathcal{M}$ has linear growth rate. But life is not that simple. Consider the class of cographic matroids. Members of this class have no $K_{5}$ minor and we have a class that is very far from being graphic, let alone embeddable on a surface. Assume then, that there is an $n$ such that $\mathcal{M}$ does not 
contain either $M\left(K_{n}\right)$ or $M\left(K_{n}\right)^{*}$. Then we have a clear answer. There is a subgroup $\Gamma$ of the multiplicative group of $G$ such that the core class is the class of Dowling matroids associated with group-labelled planar graphs where the group labels come from $\Gamma$.

We may now assume that we are dealing with a class of matroids that either contains all graphic matroids or all cographic matroids. Up to duality we may assume that $\mathcal{M}$ contains all graphic matroids. As we are in a proper minor-closed class there is some $r$ such that $P G(r-1, q)$ is not in $\mathcal{M}$. But if $k \neq 1$, so that $q$ is not prime, then the class of all $G F(q)$-representable matroids has no $P G(2, q)$-minor. It is apparent that we have to consider the subfields of $G F(q)$. For the next stage of the hierarchy we assume that there is an $r$ such that $\mathcal{M}$ does not contain all projective geometries over the base field; that is, $\mathcal{M}$ does not contain all projective geometries over $G F(p)$ where $q=p^{k}$ and $p$ is prime. In other words, there is an $r$ such that $P G(r-1, p)$ is not in $\mathcal{M}$. By the Growth Rate Theorem we are now in a class of quadratic growth rate. Moreover, it turns out that the dual of a large projective geometry has a reasonably large projective geometry as a minor. This means that the earlier complication caused by cographic matroids has disappeared. But those caused by subgroups of $G F(q)^{*}$ certainly have not. The answer now is that there is a subgroup $\Gamma$ of $G F(q)^{*}$ such that our core objects are Dowling matroids over $\Gamma$.

Finally, we may assume that we contain all $G F(p)$-representable matroids. In this case there is a subfield $\mathbb{F}$ of $G F(q)$ such that our core class is the class of $\mathbb{F}$ representable matroids. In other words, for some integer $l$ that divides $k$, the core class is the class of $G F\left(p^{l}\right)$-representable matroids.

Deviations The Graph Minors Structure Theorem allows three deviations from planarity. For the first we allow graphs to be embedded on a surface in which $K_{n}$ does not embed. That is, we allow embeddings in surfaces of bounded genus. For the second we allow a bounded number of vortices of bounded vortex width. We have no intention of discussing vortices in detail apart from observing that one can think of a vortex as a graph of bounded path width attached to a face of the graph in a way that respects the facial ordering of the vertices of attachment. Finally, we allow a bounded number of apex vertices. These are vertices attached in an arbitrary way to the surface structure. It is clear that there is a need for such vertices. Consider a very large grid with a single extra vertex attached to every vertex of the grid. This graph has no $K_{6}$ minor, but it is not embeddable over a surface of small genus, nor can it be explained through vortices. Put another way, the class of apexed planar graphs is a class with no $K_{6}$ minor that demonstrates the need for apex vertices in a structure theorem.

Since we've mentioned apexed planar graphs we'll take the opportunity to deviate for a moment from our discussion of deviations. We say that an apexed planar graph is a graph with a single apex vertex attached to a planar graph. The class of apexed planar graphs is an interesting class and arises naturally in a number of situations. Recall from the discussion on Dowling matroids, that when we take the Dowling matroid associated with a graph, we allow matroid elements on the vertices as well as the edges. If we do this with a planar graph $G$ and we perform the construction in the standard way, we obtain a matrix labelled by the vertices and edges of $G$ with the following properties. The columns labelled by the vertices form an identity matrix 
wile the remaining columns labelled by the edges of $G$ have two nonzero entries and give the cycle matroid of $G$ in the usual way. It turns out that this matroid is isomorphic to the cycle matroid of the graph obtained by adding a single apex vertex connected to every vertex of $G$. Thus, from a Dowling matroid perspective which we necessarily have in matroid structure theory - the class of apexed planar graphs is not, in itself, a deviation from planarity. Of course, if we have more than one apex vertex the situation changes.

If $\mathcal{M}$ is a class of $G F(q)$-representable matroids where we have excluded $M\left(K_{n}\right)$ and $M\left(K_{n}\right)^{*}$, then similar deviations arise from our core class. We allow group labelled graphs on surfaces of bounded genus. Vortices also inevitably arise, but we do not have graphic structure on the elements in the vortices. Nonetheless, very loosely speaking, vortices resolve as matroids of bounded path width glued to a face of the graph where the path decomposition respects the ordering of the vertices given by the face. What about apex vertices? In graphs we can think of apex vertices as adding a bounded amount of uncontrolled junk. It's clearly necessary to allow this. For matroids there is a somewhat more general way of adding a bounded amount of junk which we capture via the notion of a "perturbation". Thus, for $\mathcal{M}$, we replace adding apex vertices by performing a "bounded rank perturbation". We'll discuss perturbations more carefully in the next section.

Moving up to the higher levels in the matroid hierarchy things change. There is no road map from graphs to follow. Fortunately, as we move up, at least this aspect of structure simplifies. While we necessarily allow bounded rank perturbations, there is no higher-order analogue of surface structure or vortices. We have a single type of deviation from our core structures of Dowling matroids and matroids representable over subfields.

\section{Bounded rank perturbations}

Let $A$ and $B$ be matrices over a field $\mathbb{F}$. Then $B$ is a rank-t perturbation of $A$ if there are matrices $A^{\prime}$ and $B^{\prime}$ that are row equivalent to $A$ and $B$ respectively such that the matrix $B^{\prime}-A^{\prime}$ has rank at most $t$. Evidently, if $B$ is a rank- $t$ perturbation of $A$, then $A$ is a rank- $t$ perturbation of $B$. The perturbation distance between $A$ and $B$ is the minimum value $t$ for which $B$ is a rank- $t$ perturbation of $A$. Note that, for the perturbation distance to be defined it suffices for $A$ and $B$ to have the same number of columns as we may add rows of zeros to obtain matrices $A^{\prime}$ and $B^{\prime}$ for which $B^{\prime}-A^{\prime}$ is defined. The case we are interested in is when $A$ and $B$ represent matroids $M[A]$ and $M[B]$ on a common ground set. In this case it is straightforward to prove that a bound on the perturbation distance between $A$ and $B$ also gives a bound on the difference between the sizes of a maximum clique minor or maximum projective geometry minor of $M[A]$ and $M[B]$. In other words, bounded-rank perturbations cannot arbitrarily increase the size of such minors. Thus bounded-rank perturbations are a necessary feature of structure theory.

Let $M_{1}$ and $M_{2}$ be matroids on a common ground set. Then $M_{2}$ is a projection of $M_{1}$ if there is a matroid $M$ with a set $X$ of elements such that $M_{1}=M \backslash X$ and $M_{2}=M / X$. If $M_{2}$ is a projection of $M_{1}$, then $M_{1}$ is a lift of $M_{2}$. Moreover the rank of the projection or lift is $r\left(M_{1}\right)-r\left(M_{2}\right)$. Note that projections are often called quotients in the matroid literature; see, for example Oxley [28]. In the represented 
case projections are geometrically quite natural. In this case $E(M)$ labels a set of vectors. Now add a new vector $\mathbf{p}$ and project the points labelled by $E(M)$ from $\mathbf{p}$ onto a hyperplane that avoids $\mathbf{p}$. The resulting matroid $M^{\prime}$ on $E$ that labels the projected points is a rank- 1 projection of $M$. From a matrix perspective we have added a new column representing $\mathbf{p}$ to the matrix representing $M$ and contracted $\mathbf{p}$ in the usual way, that is, we pivot on an entry in $\mathbf{p}$ to replace it by a column with a single non-zero entry. We then delete this column and the row containing the nonzero entry. Evidently higher-rank projections can be described as a sequence of such rank-1 projections so that we are adding a set of vectors and projecting the elements of $M$ from that set.

The inverse of the above procedure describes lifts in the represented case. It probably says something about the wiring of the human brain that our geometric intuition seems more comfortable with projections than lifts. Having said that, from a matrix perspective, lifts are easily described. A rank-1 lift is obtained by adding a new row to the matrix representing $M$. Consider an example. Take a matrix over $G F(q)$ representing $M(G)$ for some graph $G$, where we have two nonzero entries in each column in the usual way. Now add an arbitrary row; the resulting matroid $M^{\prime}$ is an elementary lift of $M(G)$. It is easy to see that any perturbation can be resolved as a sequence of lifts and projections. Indeed, if $M_{1}$ and $M_{2}$ are any two represented matroids on the same ground set then they can be so resolved; simply project $M_{1}$ to a matroid consisting only of loops, encoded by a matrix of zeros, and then perform the lifts by adding the rows that we need to get a representation of $M_{2}$. We'll say that the lift-project distance between $M_{1}$ and $M_{2}$ is the minimum of the sum of the ranks of projections and lifts that transform the representation of $M_{1}$ to a representation of $M_{2}$.

We can now describe the relationship between perturbations, and lifts and projections more precisely. First observe that if the matrix $A$ represents a matroid $M$, then we may add a row to $A$ that is in the row space of $A$ and still have a representation of $M$ as we have not changed the linear independence or otherwise of sets of columns. Indeed, we may as well take the whole row space $R(A)$. Note that $R(A)$ encodes all the representations equivalent to $A$; to obtain such a representation we simply choose a set of rows containing a basis of $R(A)$.

Proposition 10.1 Let $M_{1}$ and $M_{2}$ be $\mathbb{F}$-represented matroids on a common ground set represented by $A_{1}$ and $A_{2}$ respectively. Let $R_{1}$ and $R_{2}$ denote the row space of $A_{1}$ and $A_{2}$ respectively. Let $k=\operatorname{dim}\left(R_{1} \cap R_{2}\right), k_{1}=\operatorname{dim}\left(R_{1}\right)-k$, and $k_{2}=\operatorname{dim}\left(R_{2}\right)-k$. Then the following hold.

(i) The perturbation distance between $A_{1}$ and $A_{2}$ is the maximum of $\left\{k_{1}, k_{2}\right\}$.

(ii) The lift-project distance between $A_{1}$ and $A_{2}$ is $k_{1}+k_{2}$.

Finally we consider the relationship between apex vertices and perturbations. Say that the graph $G_{2}$ is obtained from the graph $G_{1}$ by adding a set $A$ of apex vertices to $V\left(G_{1}\right)$. Now add a set $T$ of edges that forms a tree that spans the vertices in $A$. Let $G_{3}$ be the graph obtained by contracting $T$. Then $M\left(G_{3}\right)$ is a projection of $M\left(G_{2}\right)$ and $G_{3}$ can be obtained from $G_{1}$ by adding a single apex vertex. But we observed in the previous section that, in this case, $M\left(G_{3}\right)$ is a Dowling matroid that can be associated with the graph $G_{1}$. 
More on group-labelled graphs We can think of Dowling matroids as certain matroids associated with group-labelled graphs. We have already seen that these are fundamental in structure theory. The study of perturbations leads to other ways of associating matroids with group-labelled graphs that also play an important role. We will not give the full story here, but focus on the special case of binary lifts of graphic matroids. If we perform a sequence of $k$ such lifts then the resulting matroid can be represented by a matrix that is graphic in the usual way with $k$ extra rows. Each column can now be interpreted as a graph edge labelled by a $k$-tuple from the additive group of the vector space $V(k, 2)$. Our matroid now has an interpretation as a matroid associated with a group-labelled graph. This is quite distinct from the Dowling matroid that would be associated with such a group-labelled graph.

The full picture is more complex, but, associated with a perturbed Dowling matroid, there is a group-labelled graph that captures much of the crucial information of the matroid. This is important and extremely useful. On the one hand, one can generalise results for graph minors and develop structure theory for minor-closed classes of group labelled graphs, see for example [5, 22]. On the other hand, via the correspondences discussed above, it is often possible to reduce problems for matroids to questions about group-labelled graphs.

\section{Back to tangles}

The Graph Minors Structure Theorem is frequently stated in a way that makes no reference to tangles. Rather, it states that a graph can be decomposed using the operation of clique sum into parts that are almost planar. While there is an analogue of clique sum for matroids representable over finite fields, it seems unlikely that this will lead to a useful form of a structure theorem for such matroids. Rather, we focus on structure relative to a tangle in a matroid. The global structure is then understood via the structure relative to the maximal tangles together with the tree of tangles. In fact the same is true for graphs in that the strategy of the proof of the structure theorem is to focus on structure relative to a given tangle.

A reader familiar only with the definition may find a tangle a somewhat intangible thing. But there is a variety of techniques that enable one to deal with tangles. For example, there is a matroid associated with a tangle $\mathcal{T}$. But the most important fact is that grids can be connected with tangles. The grid theorem tells us that in a matroid with very high branch width, there is a large grid minor, but there is no use in having that minor in some part of the matroid that is not highly connected to the tangle - a grid in London does not give us structure in Manchester. There is a natural tangle of order $n$ that can be associated with the cycle matroid $M\left(G_{n}\right)$ of the $n \times n$ grid. Any tangle in a minor $N$ of a matroid $M$ induces a tangle in $M$ in a natural way. Thus each grid minor of $M$ induces a tangle in $M$. We say that a tangle $\mathcal{T}$ in $M$ dominates an $M\left(G_{n}\right)$-minor of $M$, if this tangle is a truncation of $\mathcal{T}$. The next theorem is proved in [10].

Theorem 11.1 For each finite field $\mathbb{F}$ and positive integer $k$, there exists an integer $\theta$ such that, if $M$ is an $\mathbb{F}$-representable matroid and $\mathcal{T}$ is a tangle in $M$ of order $\theta$, then $\mathcal{T}$ dominates an $M\left(G_{k}\right)$-minor.

This is a qualitative converse to the fact that grids have large tangles. Through 
it, structure relative to high order tangles becomes more tangible as it is, in essence, structure relative to a large grid minor. We are now in a position to give an informal statement of our structure theorem. Recall from Section 6 that certain low-rank separations are unavoidable. For graphs 3 -separations are certainly unavoidable, but we saw that for matroids certain 4-separations necessarily arise. But that is as bad as it gets. Indeed, when we get to projective geometries, it turns out that 2-separations are as bad as it gets. At times the mathematical universe is unreasonably kind to us. Let $\mathbb{F}$ be a finite field, let $\mathbb{F}_{0}$ be a subfield of $\mathbb{F}$, let $M$ be an $\mathbb{F}$-representable matroids, and let $\mathcal{T}$ be a tangle in $M$.

- For any integer $n$, there exists $\theta$ such that, if $\mathcal{T}$ has order $\theta$ in $M$ and does not control an $M\left(K_{n}\right)$ - or $M\left(K_{n}\right)^{*}$-minor, them $M$ or $M^{*}$ is essentially a frame matroid over a subgroup of $\mathbb{F}^{*}$ whose associated graph is embeddable in a surface of low genus. Here "essentially" allows low-rank perturbations, vortices and suppressing small sides of 4-separations.

- For any integer $n$, there exists $m$ such that, if $\mathcal{T}$ is a tangle in $M$ that controls an $M\left(K_{m}\right)$-minor, but does not control a $P G\left(n-1, \mathbb{F}_{0}\right)$-minor, then $M$ or $M^{*}$ is essentially a frame matroid. Here "essentially" allows low-rank perturbations and suppressing small sides of 4-separations.

- For any integer $n$, there exists $m$ such that, if $\mathcal{T}$ controls a $P G\left(m-1, \mathbb{F}_{0}\right)$-minor but does not control a $P G(n-1, \mathbb{F})$-minor, then $M$ is essentially representable over a subfield of $\mathbb{F}$. Here "essentially" allows low-rank perturbations and suppressing small sides of 2-separations.

\section{Progress towards Rota's Conjecture}

An excluded minor for minor-closed class $\mathcal{M}$ is a matroid $M$ that is not in $\mathcal{M}$ having the property that all of its proper minors belong to $\mathcal{M}$. As noted in the introduction, probably the most famous conjecture in matroid theory is

Conjecture 12.1 (Rota's Conjecture) For any finite field $G F(q)$, there are only a finite number of excluded minors for the class of $G F(q)$-representable matroids.

Applying structure theory to well-quasi-ordering and minor testing essentially follows the model established by Robertson and Seymour for graph minors. However we also expect structure theory to be a valuable tool to help resolve Rota's Conjecture. This has a somewhat different flavour and we conclude by briefly discussing how we expect structure theory to be applied to this conjecture. The following two complementary results add considerable support to Rota's Conjecture. The first is a theorem of Geelen, Gerards and Whittle [8].

Theorem 12.2 For any prime power $q$, there are only finitely many excluded minors for the class of $G F(q)$-representable matroids that contain a $P G(q+6, q)$-minor.

This is significant since it opens Rota's Conjecture to an attack via structural results on the class of $G F(q)$-representable matroids with no $P G(q+6, q)$-minor. The second is a result of Geelen and Whittle [6]. 
Theorem 12.3 For any prime power $q$ and fixed integer $k$ there are only a finite number of excluded minors for $G F(q)$-representability of branch width $k$.

This is significant since we may combine it with the grid theorem for matroids to obtain

Theorem 12.4 For any planar graph $H$ and prime power $q$, there are only a finite number of excluded minors for $G F(q)$-representability that do not contain a minor isomorphic to $M(H)$.

Thus, if Rota's Conjecture is false, then for any given planar graph $G$, there is an excluded minor containing $G$. Equivalently, there are excluded minors containing arbitrarily large grids as minors.

Even with the structure theory there are still major conceptual obstacles to overcome to be able to resolve Rota's Conjecture completely; these obstacles are largely caused by inequivalent representations of matroids and we will not go into details here. Nonetheless recent work has been promising and we are confident of a resolution of Rota's Conjecture in the not-too-distant future.

\section{References}

[1] B. Courcelle, The monadic second order logic of graphs. I. Recognizable sets of finite graphs, Information and Computation 85 (1990), 12-75.

[2] R. Diestel, T. R. Jensen, K. Yu. Gorbanov and C. Thomassen, Highly connected sets and the excluded grid theorem, J. Combin. Theory Ser. B 75 (1999), 61-73.

[3] T. A. Dowling, A class of geometric lattices based on finite groups, J. Combin. Theory Ser. B 14 (1973), 61-83.

[4] J. Geelen, Some open problems on excluding a uniform minor, Adv. in Appl. Math. 41 (2008), 628-637.

[5] J. Geelen and B. Gerards, Excluding a group labelled graph, J. Combin. Theory Ser. B 99 (2009), 247-253.

[6] J. Geelen and G. Whittle, Branch width and Rota's Conjecture, J. Combin. Theory Ser. B 86 (2002), 315-330.

[7] J. Geelen, B. Gerards and G. Whittle, Branch-width and well-quasi-ordering in matroids and graphs, J. Combin. Theory Ser. B 84 (2002), 270-290.

[8] J. Geelen, B. Gerards and G. Whittle, On Rota's Conjecture and excluded minors containing large projective geometries, J. Combin. Theory Ser. B 96 (2006), 405-425.

[9] J. Geelen, B. Gerards and G. Whittle, Excluding a planar graph from $G F(q)$ representable matroids, J. Combin. Theory Ser. B 97 (2007), 971-998.

[10] J. Geelen, B. Gerards and G. Whittle, Tangles tree-decompositions and grids in matroids, J. Combin. Theory Ser. B 99 (2009), 657-667. 
[11] J. Geelen and K. Kabell, Projective geometries in dense matroids, J. Combin. Theory Ser. B 99 (2009), 1-8.

[12] J. Geelen, J. Kung and G. Whittle, Growth rates of minor-closed classes of matroids, J. Combin. Theory Ser. B 99 (2009), 420-427.

[13] J. Geelen and G. Whittle, Cliques in dense $G F(q)$-representable matroids, J. Combin. Theory Ser. B 87 (2003), 264-269.

[14] J. Geelen and G. Whittle, Inequivalent representations of matroids over prime fields, submitted.

[15] T. Gowers, The two cultures of mathematics, in Mathematics, Frontiers and Perspectives (ed. V. Arnold, M. Atiyah, P. Lax, B. Mazur), American Mathematical Society, Rhode Island (2000).

[16] T. Gowers, Rough structure and classification, in Geom. Funct. Anal., Special Volume, Part I (2000), 79-117.

[17] I. Heller, On linear systems with integral valued solutions, Pacific J. Math. 7 (1957), 1351-1364.

[18] I. V. Hicks and N. B. McMurray, The branchwidth of graphs and their cycle matroids, J. Combin. Theory Ser. B 97 (2007), 681-692.

[19] P. Hliněný, Branch-width, parse trees and monadic second-order logic for matroids, J. Combin. Theory Ser. B 96 (2006), 325-351.

[20] P. Hliněný, Some hard problems on matroid spikes, Theory of Computing Systems 41 (2007), 551-562.

[21] P. Hliněný and G. Whittle, Matroid tree width, European J. Combin. 27 (2006), 1117-1128.

[22] T. Huynh, 2009. The linkage problem for group-labelled graphs, PhD Thesis, University of Waterloo,

[23] J. Kahn and J. P. S. Kung, Varieties of combinatorial geometries, Trans. Amer. Math. Soc. 271 (1982), 485-499.

[24] J. P. S. Kung, Extremal matroid theory, Contemporary Mathematics 147 (1993), 21-61.

[25] W. Mader, Homomorphieeigenschaften und mittlere Kantendichte von Graphen, Math. An. 174 (1967), 265-268.

[26] D. Mayhew, M. Newman and G. Whittle, On excluded minors for real representability, J. Combin. Theory Ser. B 99 (2009), 685-689.

[27] D. Mayhew, M. Newman, D. Welsh and G. Whittle, On the asymptotic proportion of connected matroids, European Journal of Combinatorics 32 (2011), $882-890$. 
[28] J. G. Oxley, Matroid Theory, Oxford University Press, Oxford (2011).

[29] J. G. Oxley, What is a matroid?, Cubo 5 (2003), 179-218.

[30] N. Robertson and P. D. Seymour, Graph Minors. V. Excluding a planar graph, J. Combin. Theory Ser. B 41 (1986), 92-114.

[31] N. Robertson and P. D. Seymour, Graph Minors. X. Obstructions to tree decomposition, J. Combin. Theory Ser. B 59 (1991), 153-190.

[32] N. Robertson and P. D. Seymour, Graph Minors. XIII. The disjoint paths problem, J. Combin. Theory Ser. B 63 (1995), 65-110.

[33] N. Robertson and P. D. Seymour, Graph Minors. XVI. Excluding a non-planar graph, J. Combin. Theory Ser. B 89 (2003), 43-76.

[34] N. Robertson and P. D. Seymour, Graph Minors. XX. Wagner's Conjecture, J. Combin. Theory Ser. B 92 (2004), 325-357.

[35] N. Robertson, P. D. Seymour and R. Thomas, Quickly excluding a planar graph, J. Combin. Theory Ser. B 62 (1994), 323-348.

[36] G. -C. Rota, Combinatorial theory, new and old, in Actes du Congres International des Mathematiciens (Nice, Sept. 1970) Tome 3, pp. 229-233, GaulthierVillars, Paris (1971).

[37] P. D. Seymour, Decomposition of regular matroids, J. Combin. Theory Ser. B 28 (1980), 305-354.

[38] P. D. Seymour, Triples in matroid circuits, European J. Combin. 7 (1986), $177-185$.

[39] P. Turán, On an extremal problem in graph theory, Matematikai és Fizikai Lapok 48 (1941), 436-452.

[40] K. Truemper, On the efficiency of representability tests for matroids, European J. Combin. 3 (1982), 275-291.

[41] W. T. Tutte, A homotopy theorem for matroids, I, II, Trans. Amer. Math. Soc. 88 (1958), 144-174.

[42] T. Zaslavsky, Biased graphs. II. The three matroids, J. Combin. Theory Ser. $B \mathbf{5 1}$ (1991), 46-72.

Department of Combinatorics and Optimization

University of Waterloo, Waterloo, Canada jfgeelen@math.uwaterloo.ca 
Centrum Wiskunde \& Informatica Amsterdam, The Netherlands

\&

Maastricht University School of Business and Economics Maastricht, The Netherlands

School of Mathematics Statistics and Operations Research Victoria University, Wellington, New Zealand

geoff.whittle@vuw.ac.nz 